RESEARCH REPORT

\title{
Long term mortality trends behind low life expectancy of Danish women
}

\section{R Jacobsen, N Keiding, E Lynge}

J Epidemiol Community Health 2002;56:205-208

See end of article for authors' affiliations

......................

Correspondence to:

Dr R Jacobsen, Institute of

Public Health, University of

Copenhagen, Blegdamsvej

3, DK-2200, Denmark;

R.jacobsen@pubhealth.ku.dk

Accepted for publication

24 July 2001

\begin{abstract}
Study objective: To examine the long time mortality trends of women in Denmark.
Design: Age-period-cohort analysis.

Setting: To search for possible causes we analysed mortality rates for all Danish women and men aged 40-84 during 1960-1999. Age-period-cohort modelling was used, handling the well known indeterminacy in a sensitivity analysis.

Main results: The results indicate that the high risk of dying among Danish women is associated with being born between the two world wars. A similar pattern was not seen for men.

Conclusion: The rather simple descriptive exploration in the framework of age-period-cohort modelling used, revealed a pattern not reflected by the commonly used life expectancy calculation. It is suggested that future studies on the low life expectancy of Danish women focus on the "between wars" generations identified with a high risk of dying in this study.
\end{abstract}

$\mathrm{T}$ he reduction of health disparities within and between countries has been described as an important issue on the public health agenda for this century. ${ }^{1}$ During the past decades, the life expectancy of Danish women has lagged behind that of women in other countries in Western Europe, USA, Canada, Australia and even recently developed countries as Costa Rica and Slovenia. The life expectancy of Danish women now ranks 36 in the world. ${ }^{2}$ The low life expectancy of Danish women has been explained by increased mortality with time among women aged 35-74 years. ${ }^{3}$ The life expectancy is an index only reflecting the mortality at a given point in time. This study was undertaken to disentangle the long term mortality changes behind the present low life expectancy of Danish women.

We used age, period, cohort models to analyse the mortality rates of Danish women born in the 20th century. If such an analysis points towards a dominant period effect then the causes for the low life expectancy should be found in a particular calendar period, whereas a dominant cohort effect would indicate causes specific to certain generations. A new approach to sensitivity analysis was used to evaluate the evidence for each of these effects. We furthermore compared the mortality pattern of Danish women with that of Danish men.

\section{METHODS}

Number of deaths and midyear population, stratified by five year groups, for all Danish women aged 40-84 in the period 1901-1999 and for men aged 40-84 in the period 1960-1999 were obtained from publications of Statistics Denmark. ${ }^{45}$ The data were organised in a two way table with rows as five year age groups and columns as five year period groups and synthetic birth cohorts represented by the diagonals of the table. Within five year age and period groups, the women contributing to such a cohort are born within a 10 year period, the same women contributing to two adjacent synthetic birth cohorts.

The period 1960-1999 were analysed using age-periodcohort modelling. ${ }^{6}$ This technique aims to solve the problem that the age, period, and cohort effects cannot be simply estimated in the same model as they are linearly dependent on each other. ${ }^{7}$ To meet this problem the linear components of age, period, and cohort and parameters associated with deviation of the effects of each of these factors from linearity can be examined. ${ }^{78}$ We used the following model to examine effects of the age, calendar period and birth cohort:

$\log \left(\right.$ rate $\left._{i j}\right)=\mu+\alpha_{i}+\beta_{i}+\epsilon_{c}$

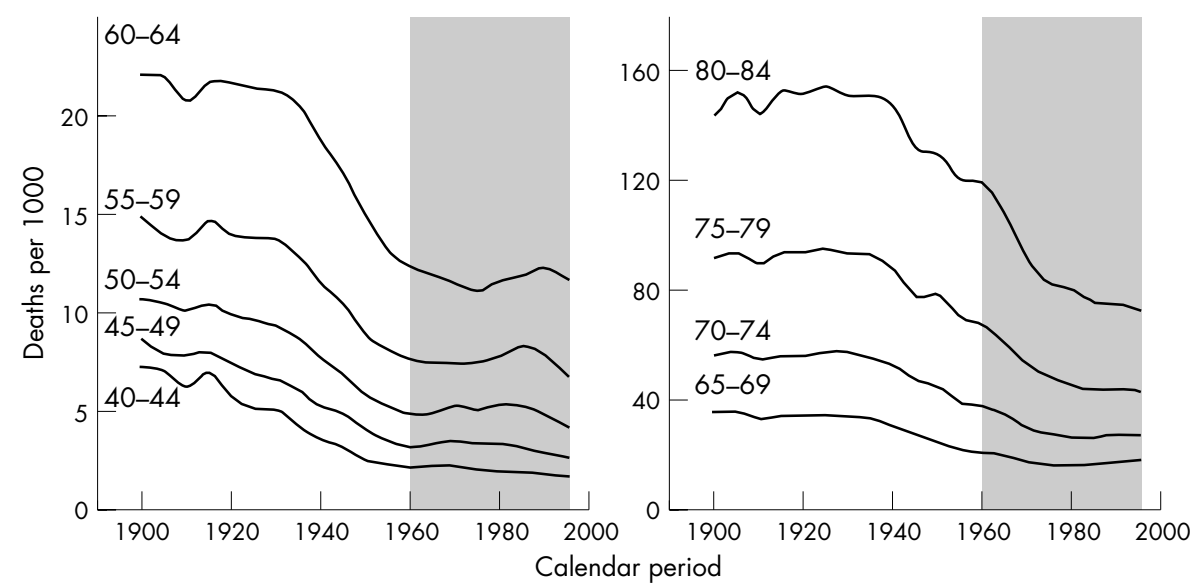

Figure 1 Mortality rates for women in Denmark by age during the 20th century. Grey area indicates period with increase in mortality rates. 

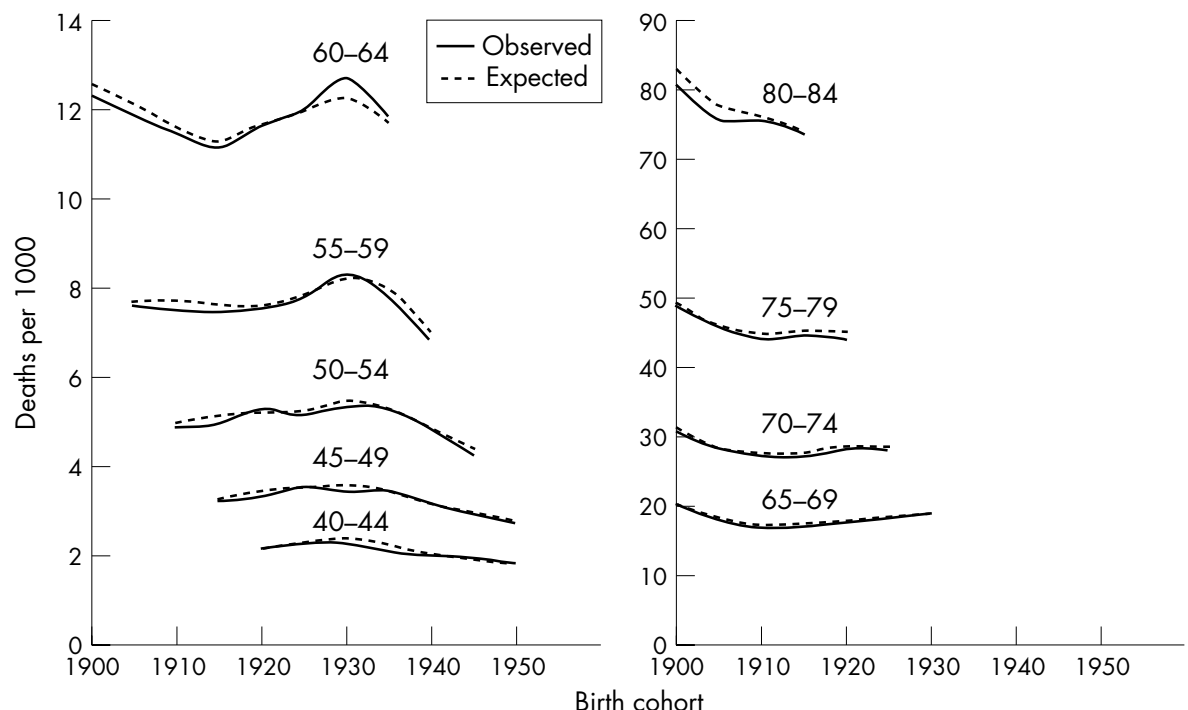

Figure 2 Observed and expected mortality rates for women in Denmark 1960-1999.

where $\mu$ represents the mean effect (intercept), $\alpha_{i}$ the effect of age group $i, \beta_{j}$ the deviation from linearity of the $j^{\text {th }}$ period, $\epsilon_{c}$ the deviation from linearity of the $c^{\text {th }}$ cohort. To validate the model we calculated the expected rates based on the model and compared them with the observed rates. Formal goodness of fit analysis, comparing the observed deviance to the relevant $\chi^{2}$ distribution, requires some care for these data based on a complete national population, as conventional significance levels are not necessarily helpful. The intrinsic indeterminacy of the linear effect (period or cohort+age) was handled in a sensitivity analysis, studying the effects of a range of plausible ways of partitioning the linear effect between the two possible sources. We assumed a five year period change in mortality rate of $0.002,0.000$, and -0.002 and examined the effect on the deviations from linearity for cohorts and periods.

The parameters of the models were estimated using multiplicative Poisson regression models ${ }^{8}$ and all statistical analyses were done using the SAS 6.12 package. $^{10}$

\section{RESULTS}

There was an overall decline in the mortality rates for Danish women during the past century (fig 1). However, around
1960-1965 the mortality rates started to increase among women aged 40-59 years, with a later rise for older age groups. Based on this observation the age, period, cohort modelling was restricted to mortality data from the period 1960-1999.

We fitted the age-period-cohort model and calculated the expected mortality rates for women. These expected rates were close to the observed mortality rates (fige 2). The model deviance was only 1.87 times the relevant degrees of freedom (36), which should be taken as a remarkably good fit for national mortality data. The modelling of the mortality data for Danish men for the period 1960-1999 gave a deviance 8.05 times the relevant degrees of freedom (36).

The relative risk of death for women and men in each age, period, and cohort group were compared with persons born in 1915-1919 and aged 50-54 in 1965-1969 (fig 3). It is essential to focus on the non-linear aspects of the relations, because (as mentioned in the method section) the linear effect cannot be uniquely ascribed either to period or cohort+age. For women, the overall cohort deviations were clearly more pronounced than the period deviations. Women born between 1920-1939 had a high relative risk of dying compared with the reference group of women born in 1915-1919. Women born in 1905-1909 and before had a high relative risk of dying
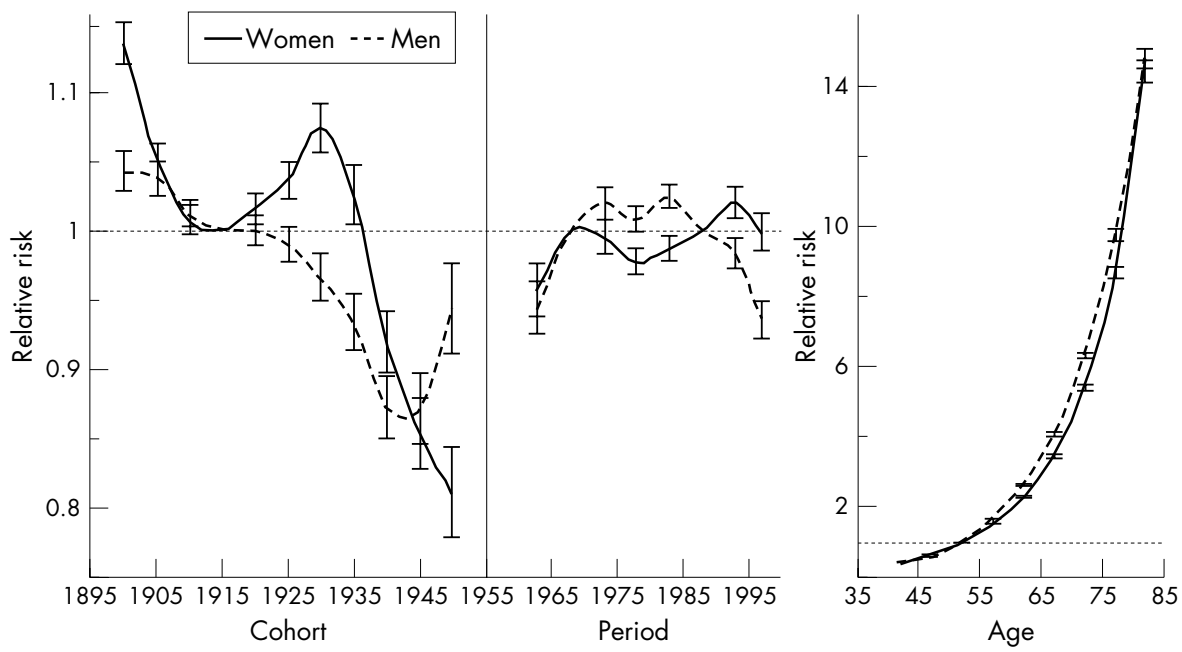

Figure 3 Relative risk of death for women and men in each age, period, and cohort group when compared with persons born in 1915-1919 and aged 50-54 in 1965-1969. The fitted piecewise constant age-period-cohort model is indicated by the 95\% confidence intervals. For increased readability this model has been represented by smoothed curves. 


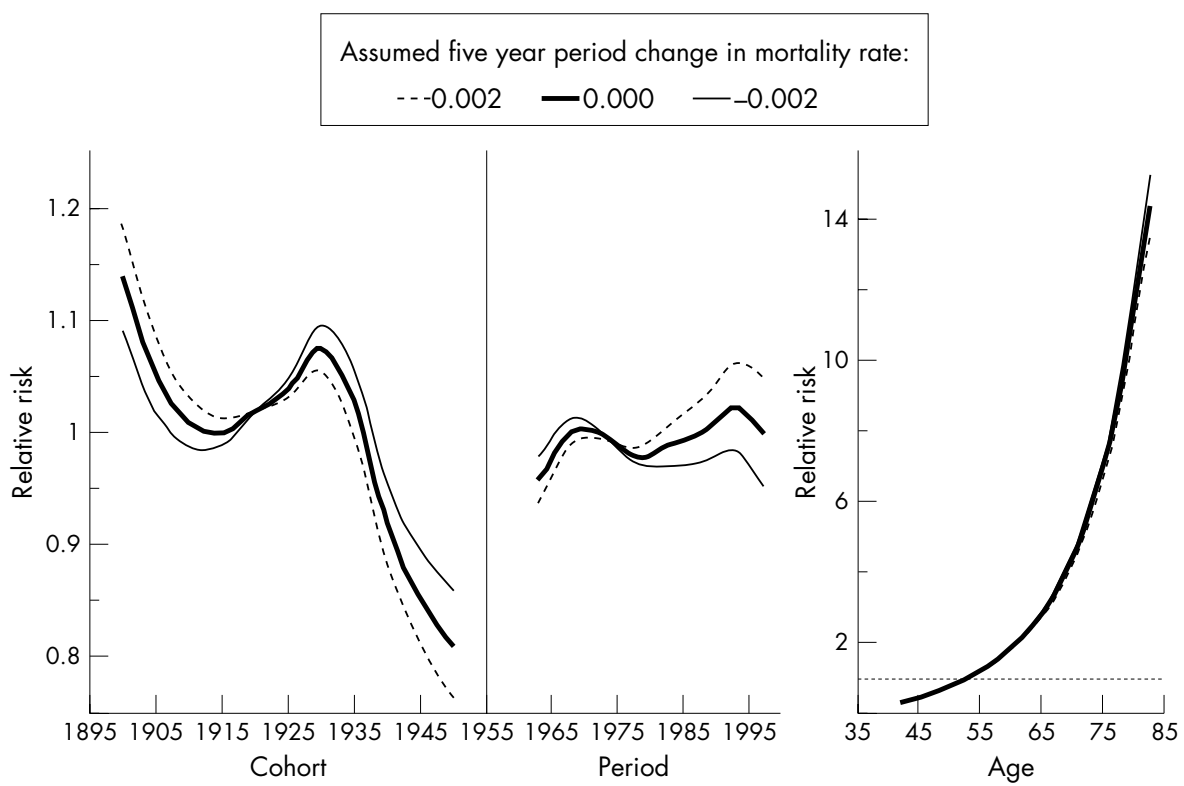

Figure 4 Effect on deviation from linearity in mortality rates for women in Denmark when partioning the linear trend between period or cohort+age. The reference group are born in 1915-1919 and aged 50-54 in 1965-1969.

\section{Key points}

- The high risk of dying among Danish women is associated with being born between the two world wars.

- We suggest that future studies focus on differences in living conditions between the high and low risk generations.

- The framework of age-period-cohort modelling presented in the study may be useful for analysis of long term mortality trends in other countries.

compared with the reference group, whereas women born in 1940 and after had a relatively low risk of dying. The deviations from linearity for calendar period showed a higher risk of dying in the period 1990-94 and a lower risk of dying in the periods 1960-1964, 1975-1979 and 1980-1984. The calendar period variations were, however, modest when compared with those observed for the cohorts of women. The clear deviation from linearity seen in the cohort effect for women was not seen for men (fig 3). For men the cohort variation seemed linear except perhaps towards the end of the period.

The sensitivity analysis showed that the marked deviations from linearity for cohorts of women born around 1930 remained when assuming different plausible values of the linear period effect (that is, decrease or increase in mortality rates with a five year increase in time) (fig 4).

\section{DISCUSSION}

Danish women have experienced an increase in mortality rates. This study suggests that higher mortality rates are associated with birth generation rather than calendar period. Among Danish women, the burden of high mortality is carried in particular by those born between the two world wars. Although Danish men also have a relatively low life expectancy, ${ }^{2}$ a similar cohort effect on the mortality was not found for men. This indicates the presence of sex specific causes behind the present mortality pattern in Denmark.

The study showed that despite the well known unidentifiability problems (mentioned in the Methods section) in age-period-cohort analysis, even a rather simple exploration in this framework yielded a more specific description of the mortality trends than the commonly used life expectancy. The finding of a relatively low survival for women being associated with birth between the wars was further supported by its robustness to plausible variations in linear period effects, in particular it remains even under a considerable positive linear trend for mortality. The goodness of fit, the sensitivity to plausible partitioning of the linear effect between period and cohort + age, and the dramatically different pattern found for women and men all contributed to the credibility of this analytical approach. Furthermore age, period, and cohort were included symmetrically in the analysis, which strongly suggests that the found cohort effect is not attributable to an artefact of the model.

A standard interpretation of a cohort effect on mortality in adult life would be an influence from conditions in fetal life. However, the missing cohort effect for men suggests the importance of factors occurring later in life and affecting women and men differently. Studies on the present causes of death in Danish women show that tobacco related causes play an important part. ${ }^{11}$ This fits well with the fact that our high risk generations of women were the first Danish women with a substantial proportion of smokers at the age of $20 .{ }^{12}$ Smoking, however, also started early in the later generations of Danish women. ${ }^{13}$ Among Danish men, the majority in both the 1920-1935 and previous generations were smokers at the age of $20 .{ }^{12}$ Our high risk generations of women were in part the mothers of the babyboomers, ${ }^{5}$ and the women most heavily hit by the epidemic of sexually transmitted diseases in the mid$1940 \mathrm{~s} .{ }^{14}$ These generations of women furthermore entered the Danish labour market in massive numbers in the 1960s. ${ }^{3}$

This study shows that birth cohort is more important than calendar period in explaining the mortality pattern of Danish women, and we suggest that future studies focus on differences in living conditions between the high and low risk generations. The framework of age-period-cohort modelling presented may be useful for understanding changes in life expectancies in other countries.

\section{Authors' affiliations}

R Jacobsen, E Lynge, Institute of Public Health, Department of Epidemiology, University of Copenhagen, Denmark

N Keiding, Department of Biostatistics, Institute of Public Health, Faculty of Health Sciences, University of Copenhagen

\section{REFERENCES}

1 Bloom BR. The future of public health. Nature 1999;402:C63-4. 
2 World Health Organisation. World Health Report 2000. Geneva: WHO, 2000

3 Sundhedsministeriet. Mortality in Denmark in the 1990s. [(In Danish]. Copenhagen: The Danish Ministry of Health, 1998.

4 Danmarks Statistik. Befolkningens Bevægelser, 1931-1999. [In Danish]. Copenhagen: 1932-2000.

5 Danmarks Statistik. Befolkningsudvikling og Sundhedsforhold 1901-60. [In Danish]. Copenhagen: Statistiske undersøgelser no 19, 1966.

6 Clayton D, Schifflers E. Models for temporal variation in cancer rates. I: Age-period and age-cohort models. Stat Med 1987;6:449-67.

7 Holford TR. Analysing the temporal effects of age, period and cohort. Stat Methods Med Res 1992;1:317-37.

8 Robertson C, Boyle P. Age-period-cohort analysis of chronic disease rates. I: Modelling approach. Stat Med 1998;17:1305-23.
9 Breslow NE, Day NE. Statistical methods in cancer research. The design and analysis of cohort studies. Vol 2. Lyon: IARC Scientific Publications no 82,1987

10 SAS. SAS/STAT Software: Changes and Enhancements through Release 6.11. Cary, NC: SAS Institute, 1996.

11 Juel K. Increased mortality among danish women: population based register study. BM 2000;321:349-50.

12 Hamtoft H, Lindhardt M. Tobacco consumption in Denmark. II. The Danish National Morbidity Survey of 1950. Cummunication no 12. Dan Med Bull 1956;3:188-96.

13 Osler M. Smoking habits in Denmark from 1953 to 1991: a comparative analysis of results from three nationwide health surveys among adult Danes in 1953-1954, 1986-1987 and 1990-1991. Int Epidemiol 1992;21:862-71.

14 Lynge $\mathbf{E}$, Jensen OM. Cohort trends in incidence of cervical cancer in Denmark in relation to gonorrheal infection. Acta Obstet Gynecol Scand 1985:64:291-6.

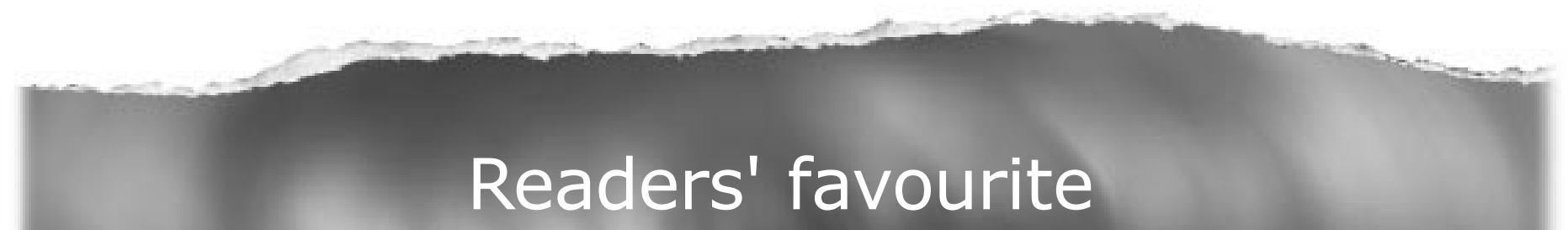

Top 10

Click on the "Top 10" button on the homepage to see which are the best read articles each month

www.jech.com 